Pacific Journal of Mathematics

ON THE MULTIPLICATIVE PROPERTIES OF ARITHMETIC 


\title{
ON THE MULTIPLICATIVE PROPERTIES OF ARITHMETIC FUNCTIONS
}

\author{
Donald L. GoldSMith
}

\begin{abstract}
In this paper we define a generalization of the set $\mathscr{C}$ of all pairs of relatively prime natural numbers and then define a limit process to measure the multiplicativity of an arithmetic function with respect to this generalized set of pairs. In so doing we gain useful information about that most important special case, namely, functions which are multiplicative in the usual sense.
\end{abstract}

2. Preliminary definitions and results. By an arithmetic function we shall mean a real-valued function $f$ whose domain is the set of natural numbers. We will deal only with arithmetic functions and, furthermore, we will assume throughout this paper that no function is eventually zero; that is, given any arithmetic function $f$ and any number $N$, there is a natural number $k \geqq N$ such that $f(k) \neq 0$.

Closely connected with the multiplicative properties of an arithmetic function is the concept of a basic sequence, which is defined as follows: A basic sequence is a set $\mathscr{B}$ of pairs of natural numbers $(a, b)$ with the following three properties:

(i) If $(a, b) \in \mathscr{B}$, then $(b, a) \in \mathscr{B}$;

(ii) $(a, b c) \in \mathscr{B}$ if and only if $(a, b) \in \mathscr{B}$ and $(a, c) \in \mathscr{B}$;

(iii) $(1, k) \in \mathscr{B}, k=1,2,3, \cdots$.

We denote by $B_{k}(k=1,2, \cdots)$ the set of pairs $(a, b) \in \mathscr{B}$ such that $a b=k$.

Let an arithmetic function $f$ and a basic sequence $\mathscr{B}$ be given. In order to measure the multiplicativity of $f$ with respect to $\mathscr{B}$ we first define

$$
\alpha_{f}(m, n)=\left\{\begin{array}{ccc}
\frac{f(m) f(n)-f(m n)}{|f(m) f(n)|+|f(m n)|} \\
0 & \text { if }|f(m) f(n)|+|f(m n)|>0, \\
& \text { if } & f(m) f(n)=f(m n)=0 .
\end{array}\right.
$$

Next we set

$$
\begin{aligned}
\bar{\alpha}(k ; f, \mathscr{B}) & =\max \left\{\alpha_{f}(m, n) \mid(m, n) \in B_{k}\right\}, \\
\underline{\alpha}(k ; f, \mathscr{B}) & =\min \left\{\alpha_{f}(m, n) \mid(m, n) \in B_{k}\right\}, \\
\bar{\alpha}(f, \mathscr{B}) & =\limsup _{k \rightarrow \infty} \bar{\alpha}(k ; f, \mathscr{B}), \\
\underline{\alpha}(f, \mathscr{B}) & =\liminf _{k \rightarrow \infty} \underline{\alpha}(k ; f, \mathscr{B}) .
\end{aligned}
$$

Finally, we define the index of multiplicativity of $f$ with respect 
to the basic sequence $\mathscr{B}$ (or, simply, the index of $f$ ) to be the common value of $\underline{\alpha}(f, \mathscr{B})$ and $\bar{\alpha}(f, \mathscr{B})$, if such a common value exists. In this case we denote the index of $f$ by $I(f, \mathscr{B})$ or, simply, by $I(f)$.

Many of the multiplicative properties of $f$ with respect to $\mathscr{B}$ will be independent of the sign of $I(f, \mathscr{B})$. Accordingly, we define

$$
\begin{aligned}
\beta_{f}(m, n) & =\left|\alpha_{f}(m, n)\right|, \\
\bar{\beta}(k ; f, \mathscr{B}) & =\max \left\{\beta_{f}(m, n) \mid(m, n) \in B_{k}\right\}, \\
\underline{\beta}(k ; f, \mathscr{B}) & =\min \left\{\beta_{f}(m, n) \mid(m, n) \in B_{k}\right\}, \\
\bar{\beta}(f, \mathscr{B}) & =\limsup _{k \rightarrow \infty} \bar{\beta}(k ; f, \mathscr{B}), \\
\beta(f, \mathscr{B}) & =\liminf _{k \rightarrow \infty} \beta(k ; f, \mathscr{B}) .
\end{aligned}
$$

$J(f, \mathscr{B})$, the absolute index of $f$, is defined to be the common value of $\bar{\beta}(f, \mathscr{B})$ and $\underline{\beta}(f, \mathscr{B})$, if such a common value exists.

When no confusion is possible we will write $I(f), J(f)$, for $I(f, \mathscr{B}), J(f, \mathscr{B})$, respectively. Occasionally we will talk about $I(f, \mathscr{B})$ or $J(f, \mathscr{B})$ without prefacing our discussion with a statement (which should then be supplied by the reader) such as "if $I(f, \mathscr{B})$ exists."

A necessary and sufficient condition that $I(f, \mathscr{B})$ exists and has the value $\varphi$ is that $\lim _{k \rightarrow \infty} \alpha_{f}\left(m_{k}, n_{k}\right)=\varphi$ for every collection of pairs $\left\{\left(m_{k}, n_{k}\right)\right\}_{k=1}^{\infty} \subset \mathscr{B}$ such that $\lim _{k \rightarrow} m_{k} n_{k}=\infty$. A similar statement holds for $J(f, \mathscr{B})$. If $I(f, \mathscr{B})$ exists, then $J(f, \mathscr{B})$ also exists and $J(f, \mathscr{B})=|I(f, \mathscr{B})|$. Without additional assumptions, however, the existence of $J(f, \mathscr{B})$ does not imply the existence of $I(f, \mathscr{B})$, except in one case: $J(f, \mathscr{B})=0$ if and only if $I(f, \mathscr{B})=0$.

EXAMPLE 2.1. Let $\mathscr{S}=\bigcup_{k=1}^{\infty} S_{k} \quad$ where $S_{k}=\{(1, k),(k, 1)\} . \quad$ If $f(1)=M>1$ and $f(k)=(-1)^{k}$ for $k>1$, then $J(f, \mathscr{S})=(M-1) /(M+1)$ but $I(f, \mathscr{S})$ does not exist.

A number of consequences follow readily from the preceding definitions.

Lemma 2.2. (a) If there exists an increasing sequence $\left\{k_{n}\right\}_{n=1}^{\infty}$ of natural numbers such that $f\left(k_{n}\right)=0$, then $\underline{\beta}(f, \mathscr{B})=0$ (in particular, if $J(f, \mathscr{B})$ exists and is not zero, then $f(k) \neq 0$ for all sufficiently large $k)$.

(b) If $\underline{\alpha}(f, \mathscr{B})>0[\bar{\alpha}(f, \mathscr{B})<0]$, then

$$
f(m) f(n)>f(m n) \quad[f(m) f(n)<f(m n)]
$$

provided $m n$ is sufficiently large and $(m, n) \in \mathscr{B}$. 
Lemma 2.3. If $\bar{\beta}(f, \mathscr{B})<1$, then there exists an integer $N$ such that, for each $k \geqq N$, exactly one of the following must hold:

(1) $f(k)=0$ and $f(m) f(n)=0$ for every $(m, n) \in B_{k}$,

(2) $f(m) f(n) \neq 0$ for any $(m, n) \in B_{k}$.

Moreover, if (2) holds, then $f(m) f(n)$ has the same sign as $f(k)$ for every $(m, n) \in B_{k}$.

Lemma 2.4. (a) $f(1) \leqq 0$ implies $\bar{\beta}(f, \mathscr{B})=1$. Thus, if $J(f)$ exists, $f(1) \leqq 0$ if and only if $J(f)=1$.

(b) Suppose $I(f)$ exists and $f(1)>1$; then $I(f)>0[I(f)<0]$ if and only if $f(k)>0[f(k)<0]$ for all sufficiently large $k$.

(c) Suppose $I(f)$ exists and $f(1)<1$; then $I(f)>0[I(f<0]$ if and only if $f(k)<0[f(k)>0]$ for all sufficiently large $k$.

Combining some of the results of the previous lemmas, we have

THEOREM 2.5. (1) If $J(f)$ exists then

$$
J(f)=\frac{|f(1)-1|}{|f(1)|+1} ;
$$

in particular, $J(f)=0$ if and only if $f(1)=1$;

(2) If $I(f)$ exists and is not zero, there is an integer $N$ such that

(a) $f(k) \neq 0$ for all $k \geqq N$, and

(b) $f(k)$ does not change sign for $k \geqq N$.

We see from Example 2.1 that part (2) above does not hold if we replace $I(f)$ by $J(f)$. Under a slightly stronger hypothesis we are able to determine the sign of $f(k)$ for large values of $k$.

Lemma 2.6. If $f(k)<0$ for all $k \geqq N$ and $\bar{\beta}(f, \mathscr{B})<1$, then $\mathscr{B}=\mathscr{S}$.

Proof. The lemma follows easily from Lemma 2.3.

As an immediate consequence of this lemma and Theorem 2.5(2) we have

CoRollary 2.6.1. Suppose that $0<|I(f, \mathscr{B})|<1$. If $\mathscr{B} \neq \mathscr{S}$, then $f(k)>0$ for all sufficiently large $k$.

EXAMPLE 2.7. Let $\mathscr{B}$ be the basic sequence consisting of $\mathscr{S}$ together with all pairs of the form $\left(2^{a}, 3^{b}\right), a \geqq 1, b \geqq 1$. Let $f(k)=$ $M(M>0, M \neq 1)$ for $k=2^{a} 3^{b}, a \geqq 0, \quad b \geqq 0$, and let $f(k)=-1$ otherwise. Then $\mathscr{B} \neq \mathscr{S}$ and $0<J(f, \mathscr{B})<1$, but $f(k)$ keeps changing sign. Hence Corollary 2.6.1 is not valid with $I(f, \mathscr{B})$ 
replaced by $J(f, \mathscr{B})$.

The next lemma and the theorem following, which will conclude this section, relate the existence of $J(f, \mathscr{B})$ to the behavior of $f(m) f(n) / f(m n)$. We will deal with sequences $\left\{\rho_{\nu}\right\}_{\nu=1}^{\infty}$ where

$$
\begin{array}{ll}
\rho_{\nu}=\frac{f\left(m_{\nu}\right) f\left(n_{\nu}\right)}{f\left(m_{\nu} n_{\nu}\right)}, & \left(m_{\nu}, n_{\nu}\right) \in \mathscr{B}, \\
& \lim _{\nu \rightarrow \infty} m_{\nu} n_{\nu}=\infty, \quad f\left(m_{\nu} n_{\nu}\right) \neq 0 .
\end{array}
$$

LEMmA 2.8. If $\bar{\beta}(f, \mathscr{B})<1$ there exist constants $N, M_{1}, M_{2}$ such that

$$
0<M_{1}<\frac{f(m) f(n)}{f(m n)}<M_{2}
$$

whenever $(m, n) \in \mathscr{B}, m n \geqq N, f(m n) \neq 0$.

THeOREM 2.9. If (1) $f(k) \neq 0$ for $k \geqq N$ and (2) if every sequence $\left\{\rho_{\nu}\right\}$ satisfying (2.3) may be decomposed into two subsequences $\left\{\varphi_{\nu}\right\},\left\{\psi_{\nu}\right\}$ (one of which may be empty) such that

$$
\lim _{\nu \rightarrow \infty} \varphi_{\nu}=f(1), \quad \lim _{\nu \rightarrow \infty} \psi_{\nu}=\frac{1}{f(1)},
$$

then $J(f, \mathscr{B})$ exists. Conversely, if $J(f, \mathscr{B})$ exists and $J(f, \mathscr{B})<1$, then every sequence $\left\{\rho_{\nu}\right\}$ satisfying (2.3) allows $a$ decomposition (where, again, one subsequence may be empty) such that (2.4) holds.

Proof. The first part of the theorem follows directly from definitions (2.2), the second part from Lemma 2.4, Theorem 2.5, and Lemma 2.8 .

3. The basic sequence. It is clear that the size of the basic sequence $\mathscr{B}$ will play a crucial role in the existence of $I(f, \mathscr{B})$. For example, $I(f, \mathscr{S})$ will exist (and have the value 0 ) whenever $f(1)=1$, while at the other extreme, the existence of $I(f, \mathscr{L})$, where $\mathscr{L}$ is the basic sequence consisting of all pairs $(m, n)$ of natural numbers, imposes severe demands upon $f$.

For any finite set $S$, let us denote by $\# S$ the number of distinct elements in $S$. In order to make more precise the idea of "size" of a basic sequence $\mathscr{B}$, we make the following definitions: For a basic sequence $\mathscr{B} \equiv \bigcup_{k=1}^{\infty} B_{k}$, set

$$
\delta_{n}(\mathscr{B})=\frac{1}{n} \sum_{k=1}^{n} \frac{\# B_{k}}{d(k)},
$$


where $d(k)$ represents the number of divisors of $k$,

$$
\begin{aligned}
& \bar{\delta}(\mathscr{B})=\limsup _{n \rightarrow \infty} \delta_{n}(\mathscr{B}), \\
& \underline{\delta}(\mathscr{B})=\liminf _{n \rightarrow \infty} \delta_{n}(\mathscr{B}) .
\end{aligned}
$$

$\bar{\delta}(\mathscr{B})$ and $\underline{\delta}(\mathscr{B})$ will be called the upper density and lower density of $\mathscr{B}$, respectively. If the upper and lower densities have a common value, we will define the density of $\mathscr{B}$ to be that common value, and denote it by $\delta(\mathscr{B})$.

EXAMPLE 3.1. Let $\mathscr{C} \equiv \bigcup_{k=1}^{\infty} M_{k}$ be the basic sequence consisting of all pairs of natural numbers $(a, b)$ for which $a$ and $b$ are relatively prime. We assert that $\underline{\delta}(\mathscr{l}) \geqq 6 / \pi^{2}$. It is clear that $M_{k}=2^{\omega(k)}$ where $\omega(k)$ is the number of distinct prime divisors of $k$, and so ${ }^{*} M_{k}=d(k)$ whenever $k$ is square-free. By [1, Th. 333], the number of squarefree integers not exceeding $x$ is $6 x / \pi^{2}+O(\sqrt{x})$. Hence

$$
\sum_{k=1}^{n} \frac{\# M_{k}}{d(k)} \geqq \sum_{\substack{k \leqq n \\ k \text { square-free }}} 1=6 n / \pi^{2}+O(\sqrt{n}),
$$

from which the assertion follows.

The above estimate for $\delta(\mathscr{l l})$ is considerably too low and will be improved shortly.

For any set $S$ of real numbers, let us define $S(k)=\{x \mid x \in S, x \leqq k\}$.

LEMmA 3.2. If $\left\{b_{k}\right\}$ is a bounded sequence of real numbers, then

$$
\lim _{n \rightarrow \infty} \frac{1}{n} \sum_{k=1}^{n} \frac{b_{k}}{d(k)}=0 \text {. }
$$

In particular, if ${ }^{\sharp} B_{k}<M(k=1,2,3, \cdots)$, then $\delta(\mathscr{B})=0$.

Proof. For a fixed $\varepsilon, 0<\varepsilon<1$, let $A$ be the set of positive integers $k$ for which it is not true that $2^{(1-\varepsilon) \log \log k}<d(k)<2^{(1+\varepsilon) \log \log k}$. By $[1$, p. 359, Th. 432$],{ }^{\sharp} A(n)=o(n)$. Hence

$$
\begin{aligned}
\frac{1}{n} \sum_{k \leqq n} \frac{1}{d(k)} & =\frac{1}{n}\left(\sum_{\substack{k \leq n \\
k \notin A}} \frac{1}{d(k)}+\sum_{k \in A(n)} \frac{1}{d(k)}\right) \\
& \leqq \frac{1}{n} \sum_{\substack{k \leq n \\
k \notin A}} 2^{-(1-\varepsilon) \log \log k}+\frac{\# A(n)}{2 n} \\
& =\frac{1}{n} \sum_{\substack{k \leq n \\
k \notin A}}(\log k)^{-(1-\varepsilon) \log 2}+o(1) .
\end{aligned}
$$

The lemma now follows from the fact that if $c>0$, 


$$
\sum_{\substack{k \leq n \\ k \notin A}}(\log k)^{-c} \leqq \int_{3}^{n}(\log x)^{-c} d x+O(1)=O\left(n(\log n)^{-c}\right) .
$$

From Corollary 2.6.1. and the above lemma we have

Corollary 3.2.1. If $0<|I(f, \mathscr{B})|<1$ and $\bar{\delta}(\mathscr{B})>0$, then $f(k)>0$ for all sufficiently large $k$.

EXAMPLE 3.3. Let $\mathscr{T}_{m} \equiv \bigcup_{k=1}^{\infty} T_{k}$ consist of the basic sequence $\mathscr{S}$ together with all pairs of the form $\left(m^{a}, m^{b}\right), a \geqq 1, b \geqq 1$. Then ${ }^{\#} T_{m^{a}}=a+1$ but $\delta\left(\mathscr{T}_{m}\right)=0$. Hence the boundedness of $\left\{{ }^{\sharp} B_{k}\right\}$ is sufficient to make $\delta(\mathscr{B})=0$, but it is not necessary.

Let us turn next to a more detailed discussion of the relationship between the structure of a basic sequence $\mathscr{B}$ and its density. Suppose that $\Phi$ is a set of pairs of natural numbers. We define the basic sequence generated by $\Phi$ to be $\Gamma(\Phi)=\bigcap \mathscr{E}$ where the intersection is taken over all basic sequences $\mathscr{E}$ which contain $\Phi$. We will call a pair $(m, n)$ a primitive pair if both $m$ and $n$ are primes. If $m \neq n$, then the primitive pair $(m, n)$ is said to be of type I, otherwise it is a primitive pair of type II.

Suppose that we represent $m$ and $n$ canonically: $m=p_{1}^{a_{1}} p_{2}^{a_{2}} \cdots p_{r}^{a_{r}}$, $n=q_{1}^{b_{1}} q_{2}^{b_{2}} \cdots q_{s}^{b_{s}}$. Then it is clear that $(m, n)$ is a member of $\mathscr{B}$ if and only if the primitive pairs $\left(p_{i}, q_{j}\right)$ are all in $\mathscr{B}(i=1, \cdots, r ; j=$ $1, \cdots, s)$. Therefore, a basic sequence $\mathscr{B}$ is completely determined by its subset of primitive pairs: $\mathscr{B}=\Gamma(\mathscr{B} \cap \Delta)$, where $\Delta$ represents the set of all primitive pairs. In view of the preceding remark it seems reasonable to expect that we should be able to make a statement about $\delta(\mathscr{B})$ if we have enough information about the primitive pairs in $\mathscr{B}$. To pursue this further we need some preliminary results.

Let $A$ be a set of distinct natural numbers. We define the asymptotic density of $A$ to be $\underline{D}(A)=\liminf _{n \rightarrow \infty} " A(n) / n$. If $* A(n) / n$ has a limit as $n \rightarrow \infty$, we call that limit the natural density of $A$ and denote it by $D(A)$. The following lemma is a standard result (see, for example, [2] p. 228-230) which we state here for convenience.

LemMA 3.4. Let $N$ be a fixed positive integer. If each member of $A$ has $N$ or fewer distinct prime factors, then $D(A)=0$.

We will say that a prime $p$ is finitely distributed with respect to a basic sequence $\mathscr{B}$ if $p$ appears as a co-member in only a finite number of primitive pairs of $\mathscr{B}$. Also, $p$ is said to have property $F$ with respect to $\mathscr{B}$ if

(1) $p$ is finitely distributed with respect to $\mathscr{B}$, and

(2) every prime $q$ with which $p$ appears as a co-member of a primitive pair in $\mathscr{B}$ is also finitely distributed with respect to $\mathscr{B}$. 
THEOREM 3.5. If there is a finite set $\left\{p_{1}, p_{2}, \cdots, p_{M}\right\}$ of distinct primes each of which has property $F$ with respect to the basic sequence $\mathscr{B}$, then

$$
\bar{\delta}(\mathscr{B}) \leqq \prod_{i=1}^{M}\left(1-\frac{1}{p_{i}}\right)
$$

Proof. We will prove the theorem for the case when $M=2$, since this contains all the essentials of the general case. First we write

$$
\frac{1}{n} \sum_{k=1}^{n} \frac{{ }^{\sharp} B_{k}}{d(k)}=\frac{1}{n}\left(\Sigma_{1}+\Sigma_{2}+\Sigma_{3}\right) \frac{{ }^{\sharp} B_{k}}{d(k)},
$$

where $\Sigma_{1}$ is taken over all $k \leqq n$ such that ${ }^{\sharp} B_{k}=2$ and either $p_{1} \mid k$ or $p_{2} \mid k ; \Sigma_{2}$ is taken over those $k \leqq n$ for which ${ }^{\sharp} B_{k}>2$ and such that either $p_{1} \mid k$ or $p_{2} \mid k$; and $\Sigma_{3}$ is taken over the remaining $k \leqq n$.

By Lemma 3.2, $\Sigma_{1}{ }^{\sharp} B_{k} / d(k)=o(n)$. To estimate $\Sigma_{2}$ we let $A$ represent the set of all positive integers $t$ for which ${ }^{\sharp} B_{t}>2$ and either $p_{1} \mid t$ or $p_{2} \mid t$. For a fixed member $k$ in $A$, there are integers $m$ and $n$ with $m>1, n>1, m n=k$ and $(m, n) \in \mathscr{B}$. Since either $p_{1}$ or $p_{2}$ divides $m n$, let us assume that $p_{1} \mid m$. Suppose that $q_{1}, q_{2}, \cdots, q_{r}$ are the only primes that appear as a co-member with $p_{1}$ in a pair in $\mathscr{B}$. Then $n=q_{1}^{a_{1}} q_{2}^{a_{2}} \cdots q_{r}^{a_{r}}, a_{i} \geqq 0(i=1,2, \cdots, r)$, and not all the $a_{i}$ are zero. Suppose also that there are $N_{i}$ primes which appear as co-member with $q_{i}(i=1,2, \cdots, r)$. If a prime $q$ divides $m$, then $q$ is a co-member with $q_{i}$ for at least one $1 \leqq i \leqq r$. Hence there are at most $N_{1}+$ $N_{2}+\cdots+N_{r}$ possible choices for $q$ and therefore not more than $r+N_{1}+N_{2}+\cdots+N_{r}$ distinct prime factors in $k$. Similarly, if $p_{2} \mid k$, we again get an upper bound for the number of distinct prime factors of $k$. It follows then from Lemma 3.4 that $D(A)=0$. Hence

$$
\lim _{n \rightarrow \infty} \frac{1}{n} \Sigma_{2} \frac{\# B_{k}}{d(k)}=\lim _{n \rightarrow \infty} \frac{1}{n} \sum_{k \in A(n)} \frac{\# B_{k}}{d(k)} \leqq \lim _{n \rightarrow \infty} \frac{\# A(n)}{n}=0 .
$$

Finally, since ${ }^{\sharp} B_{k} \leqq d(k)$,

$$
\Sigma_{3} \frac{\# B_{k}}{d(k)} \leqq \sum_{\substack{k \leq n \\ p_{1} \nmid k, p_{2} \nmid k}} 1=n-\left(\left[\frac{n}{p_{1}}\right]+\left[\frac{n}{p_{2}}\right]-\left[\frac{n}{p_{1} p_{2}}\right]\right) .
$$

Therefore

$$
\begin{aligned}
\bar{\delta}(\mathscr{B}) & =\limsup _{n \rightarrow \infty} \frac{1}{n} \sum_{k \leqq n} \frac{{ }^{\sharp} B_{k}}{d(k)} \\
& \leqq \limsup _{n \rightarrow \infty}\left\{1-\frac{1}{n}\left(\left[\frac{n}{p_{1}}\right]+\left[\frac{n}{p_{2}}\right]-\left[\frac{n}{p_{1} p_{2}}\right]\right)\right\} \\
& =\left(1-\frac{1}{p_{1}}\right)\left(1-\frac{1}{p_{2}}\right)
\end{aligned}
$$


which proves $(3.1)$.

COROLlaRY 3.5.1. If $\left\{p_{i}\right\}$ is a sequence of distinct prime numbers such that

(i) $\Sigma p_{i}^{-1}$ diverges,

(ii) $p_{i}$ has property $F$ with respect to a basic sequence $\mathscr{S}$, then $\delta(\mathscr{B})=0$.

COROLlARY 3.5.2. If $\mathscr{B}$ is a basic sequence such that every prime $p$ is finitely distributed with respect to $\mathscr{B}$, then $\delta(\mathscr{B})=0$.

CoRollary 3.5.3. If $\Phi$ consists of

(a) a finite number of primitive pairs of type $I$, and

(b) any collection of primitive pairs of type $I I$, then $\delta(\Gamma(\Phi))=0$.

If we impose a restriction somewhat more severe than property $F$ on the primes in Theorem 3.5, then the possibility of inequality in (3.1) can be eliminated. In particular, we have

THEOREM 3.6. If $\left\{p_{1}, \cdots, p_{M}\right\}$ is a finite collection of distinct primes, let $\mathscr{B}\left[p_{1}, p_{2}, \cdots, p_{M}\right]$ denote the basic sequence $\mathscr{B} \equiv \bigcup_{k=1}^{\infty} B_{k}$ such that

(i) ${ }^{\sharp} B_{k}=2$ if $p_{i} \mid k$ for at least one $1 \leqq i \leqq M$, and

(ii) ${ }^{\sharp} B_{k}=d(k)$ if $p_{i} \nmid k, i=1,2, \cdots, M$.

Then

$$
\delta\left(\mathscr{B}\left[p_{1}, \cdots, p_{M}\right]\right)=\prod_{i=1}^{M}\left(1-\frac{1}{p_{i}}\right) .
$$

Proof. We remark first that condition (i) is equivalent to the fact that $p_{i}(i=1, \cdots, M)$ does not appear in any primitive pair in $\mathscr{B}$, certainly a severe form of property $F$.

To prove the theorem we merely note that the inequality in (3.2) becomes an equality.

COROLLARY 3.6.1. For any $0 \leqq \gamma \leqq 1$, there is a sequence $\left\{\mathscr{B}_{m}\right\}_{m=1}^{\infty}$ of basic sequences such that $\lim _{m \rightarrow \infty} \delta\left(\mathscr{B}_{m}\right)=\gamma$.

$$
\text { Proof. If } \gamma=1 \text {, let } \mathscr{B}_{m}=\mathscr{L}(m=1,2, \cdots) \text {. If } 0 \leqq \gamma<1 \text {, }
$$
choose a sequence of distinct primes $\left\{p_{i}\right\}$ such that

$$
\lim _{m \rightarrow \infty} \prod_{i=1}^{m}\left(1-\frac{1}{p_{i}}\right)=\gamma \text {, and let } \mathscr{S}_{m}=\mathscr{B}\left[p_{1}, p_{2}, \cdots, p_{m}\right] \text {. }
$$

Theorem 3.6 treats the question: what happens if each member of a finite set of primes appears in no primitive pair in $\mathscr{B}$ ? We may relax this restriction somewhat and ask: what happens if each member of a finite set of primes appears in no type II primitive pair in $\mathscr{B}$ ? 
The answer to this question is given by

THEOREM 3.7. If $p_{1}, p_{2}, \cdots, p_{N}$ are distinct primes such that $\left(p_{i}, p_{i}\right) \notin \mathscr{B}, i=1,2, \cdots, N$, then

$$
\bar{\delta}(\mathscr{B}) \leqq \prod_{i=1}^{N}\left(1-\lambda_{p_{i}}\right) \text {, }
$$

where

$$
\lambda_{x}=2-\frac{1}{x}+2 x\left(1-\frac{1}{x}\right) \log \left(1-\frac{1}{x}\right) \quad \text { for } x>1 .
$$

Proof. We shall prove the theorem for the case $N=2$, since once again this contains all the essentials of the general case.

Let $p_{1}=p$ and $p_{2}=q$. If $(p, p)$ and $(q, q)$ are not in $\mathscr{B}$, then certainly $(p s, p t)$ and $(q s, q t)$ are also not in $\mathscr{B}$ for any natural numbers $s$ and $t$. Hence, if $p^{2} \mid k$, there are $d\left(k / p^{2}\right)$ pairs $(p s, p t)$ with $p^{2} s t=k$, and so

$$
{ }^{\sharp} B_{k} \leqq d(k)-d\left(k / p^{2}\right) \text {. }
$$

It follows then that

$$
\begin{aligned}
\bar{\delta}(\mathscr{B})= & \limsup _{n \rightarrow \infty} \frac{1}{n} \sum_{k=1}^{n} \frac{\# B_{k}}{d(k)} \\
\leqq & \limsup _{n \rightarrow \infty}\left(1-\frac{1}{n} \sum_{\nu \leqq n / p^{2}} \frac{d(\nu)}{d\left(p^{2} \nu\right)}-\frac{1}{n} \sum_{\nu \leqq n / q^{2}} \frac{d(\nu)}{d\left(q^{2} \nu\right)}\right. \\
& \left.+\frac{1}{n} \sum_{\nu \leqq n / p^{2} q^{2}} \frac{d(\nu)}{d\left(p^{2} q^{2} \nu\right)}\right),
\end{aligned}
$$

where we have used the fact that any pair counted in both the first and second sums must be of the form (pqs, pqt).

Now if we mean by $a^{t}|| b$ that $a^{t} \mid b$ but $a^{t+1} \nmid b$, then

$$
\sum_{\nu \leqq n \mid p^{2}} \frac{d(\nu)}{d\left(p^{2} \nu\right)}=\sum_{i=0}^{K} \sum_{\substack{\nu \leq n\left|p^{2} \\ p^{i}\right| \mid \nu}} \frac{d(\nu)}{d\left(p^{2} \nu\right)}
$$

where $K=[\log n / \log p]-2$. If $\nu=p^{i} w$ where $p \nmid w$, we have

Therefore

$$
\frac{d(\nu)}{d\left(p^{2} \nu\right)}=\frac{d\left(p^{i}\right)}{d\left(p^{i+2}\right)}=\frac{i+1}{i+3} \text {. }
$$

$$
\begin{aligned}
\sum_{\nu \leqq n \mid p^{2}} \frac{d(\nu)}{d\left(p^{2} \nu\right)} & =\sum_{i=0}^{K} \frac{i+1}{i+3} \sum_{\substack{\nu \leqq n\left|p^{2} \\
p^{i}\right| \mid \nu}} 1 \\
& =\sum_{i=0}^{K} \frac{i+1}{i+3}\left(\frac{n}{p^{i+2}}-\frac{n}{p^{i+3}}+O(1)\right) \\
& =\frac{n}{p^{2}}\left(1-\frac{1}{p}\right) \sum_{i=0}^{K}\left(\frac{i+1}{i+3}\right) \frac{1}{p^{i}}+O(\log n) .
\end{aligned}
$$


Thus, since

$$
\sum_{i=0}^{\infty}\left(\frac{i+1}{i+3}\right) x^{i}=\frac{2-x}{x^{2}(1-x)}+\frac{2}{x^{3}} \log (1-x)
$$

if $0<|x|<1$, and since

$$
\sum_{i=K+1}^{\infty}\left(\frac{i+1}{i+3}\right) \frac{1}{p^{i}}=O\left(\frac{1}{n}\right)
$$

we have

$$
\begin{aligned}
\sum_{\nu \leqq n / p^{2}} \frac{d(\nu)}{d\left(p^{2} \nu\right)}= & \frac{n}{p^{2}}\left(1-\frac{1}{p}\right)\left\{( 2 - \frac { 1 } { p } ) \left(\frac{1}{p^{2}}\left(1-\frac{1}{p}\right)\right.\right. \\
& \left.+2 p^{3} \log \left(1-\frac{1}{p}\right)+O\left(\frac{1}{n}\right)\right\}+O(\log n) \\
= & n \lambda_{p}+O(\log n) .
\end{aligned}
$$

Relation (3.7) still remains valid, of course, if we replace $p$ by $q$.

Next, by reasoning similar to that used above, we may show that

$$
\sum_{\nu \leqq n / p 2 q 2} \frac{d(\nu)}{d\left(p^{2} q^{2} \nu\right)}=n \lambda_{p} \lambda_{q}+O\left(\log ^{2} n\right) .
$$

Relation (3.4) now follows from relations (3.6)-(3.8), and so the proof is complete.

REMARK 3.8. With methods like those used in the above theorem, one may gain similar results for type I primitive pairs. One may show, for example, that if $(p, q) \notin \mathscr{B}$ for distinct primes $p$ and $q$, then

$$
\bar{\delta}(\mathscr{B}) \leqq 1-\frac{1}{2}\left(\lambda_{p}+\frac{1}{p}\right)\left(\lambda_{q}+\frac{1}{q}\right)+\lambda_{p} \lambda_{q} .
$$

Thus, since $\bar{\delta}(\mathscr{B})<1$ whenever any primitive pair is missing from $\mathscr{B}$, we have

TheOREM 3.9. $\bar{\delta}(\mathscr{B})=1$ if and only if $\mathscr{B}=\mathscr{L}$.

Just as we were able to sharpen Theorem 3.5 when we knew exactly which primitive pairs were in $\mathscr{B}$, the same knowledge allows us to improve Theorem 3.7. To be precise, we have

THEOREM 3.10. (a) If $\left(p_{1}, p_{1}\right), \cdots,\left(p_{N}, p_{N}\right)$ are the only primitive pairs not in $\mathscr{B}$, then

$$
\delta(\mathscr{B})=\prod_{i=1}^{N}\left(1-\lambda_{p}\right) .
$$

(b) If $\left\{\left(p_{i}, p_{i}\right)\right\}_{i=1}^{\infty}$ are the only primitive pairs not in $\mathscr{B}$, then 


$$
\delta(\mathscr{B})=\prod_{i=1}^{\infty}\left(1-\lambda_{p_{i}}\right) .
$$

In particular, $\delta(\mathscr{C l})=\Pi_{p}\left(1-\lambda_{p}\right) \approx 0.8191+$.

Proof. Part (a) follows immediately from the fact that relation (3.6) becomes an equality if $(p, p)$ and $(q, q)$ are the only primitive pairs not in $\mathscr{B}$.

To prove (b), let us denote by $\mathscr{B}^{(x)} \equiv \bigcup_{k=1}^{\infty} B_{k}^{(x)}$ the basic sequence generated by the set of all primitive pairs except those $\left(p_{i}, p_{i}\right)$ for which $p_{i}<x$. Clearly

$$
\bar{\delta}(\mathscr{B}) \leqq \bar{\delta}\left(\mathscr{B}^{(x)}\right)=\prod_{p_{i}<x}\left(1-\lambda_{p_{i}}\right)
$$

for any $x$. If we let $x \rightarrow \infty$ in (3.9), then we have

$$
\bar{\delta}(\mathscr{B}) \leqq \prod_{i=1}^{\infty}\left(1-\lambda_{p_{i}}\right) \text {, }
$$

On the other hand, suppose that $N$ is any fixed positive integer and that $n>N$. We may write

$$
\underline{\delta}(\mathscr{B})=\liminf _{n \rightarrow \infty} \frac{1}{n} \sum_{k \leqq n} \frac{\# B_{k}}{d(k)}=\liminf _{n \rightarrow \infty} \frac{1}{n}\left(\Sigma_{1}+\Sigma_{2}\right) \frac{\# B_{k}}{d(k)},
$$

where $\Sigma_{1}$ is taken over those $k \leqq n$ such that $p_{i}^{2} \nmid k$ for any $p_{i} \geqq N$; $\Sigma_{2}$ is taken over those $k \leqq n$ such that $p_{i}^{2} \mid k$ for at least one $p_{i} \geqq N$.

Now if $k$ is counted by $\Sigma_{1}$, then ${ }^{\sharp} B_{k}={ }^{\sharp} B_{k}^{(N)}$. Moreover, since each integer counted by $\Sigma_{2}$ is divisible by the square of a prime $p \geqq N$, the number of integers counted by $\Sigma_{2}$ is certainly not more than

$$
\frac{n}{N^{2}}+\frac{n}{(N+1)^{2}}+\frac{n}{(N+2)^{2}}+\cdots=O\left(\frac{n}{N}\right) \text {. }
$$

Hence

$$
\begin{aligned}
\underline{\delta}(\mathscr{B}) & =\liminf _{n \rightarrow \infty} \frac{1}{n}\left(\Sigma_{1} \frac{{ }^{\#} B_{k}^{(N)}}{d(k)}+\Sigma_{2} \frac{\# B_{k}}{d(k)}\right) \\
& \geqq \liminf _{n \rightarrow \infty} \frac{1}{n} \sum_{k \leqq n} \frac{\# B_{k}^{(N)}}{d(k)}+O\left(\frac{1}{N}\right) \\
& =\prod_{p_{i}<N}\left(1-\lambda_{p_{i}}\right)+O\left(\frac{1}{N}\right) .
\end{aligned}
$$

If we let $N \rightarrow \infty$, we have

$$
\underline{\delta}(\mathscr{B}) \geqq \prod_{i=1}^{\infty}\left(1-\lambda_{p_{i}}\right) .
$$

The theorem now follows from (3.10) and (3.11). 
4. Nonsingular functions. An arithmetic function $f$ will be called nonsingular with respect to a basic sequence $\mathscr{B}$ if $I(f, \mathscr{B})$ exists and is zero; the set of all such functions will be denoted by $N(\mathscr{B})$. All other functions will be called singular with respect to $\mathscr{B}$. We shall fix our attention on certain subsets of $N(\mathscr{B})$ :

(1) An arithmetic function $f$ is said to be eventually multiplicative with respect to $\mathscr{B}, f \in E M(\mathscr{B})$, if there is an integer $N$ such that $f(m) f(n)=(f m n)$ whenever $(m, n) \in \mathscr{B}$ and $m n \geqq N$. When we wish to emphasize the role of the integer $N$, we will write $f \in E M(\mathscr{B}, N)$.

(2) An arithmetic function $f$ is said to be multiplicative with respect to $\mathscr{B}, f \in M(\mathscr{B})$, if $f(m) f(n)=f(m n)$ for every $(m, n) \in \mathscr{B}$.

We note that $f$ is multiplicative in the usual sense if $f \in M(\mathscr{C})$ and that $f$ is a character if $f \in M(\mathscr{L})$. Since $f(1)=1$ if $f \in N(\mathscr{B})$, it is clear that the sets $E M(\mathscr{B}, N)$ and $M(\mathscr{B})$ are identical if $N=1,2,3$, or 4 .

Since $M(\mathscr{B}) \subset E M(\mathscr{B}) \subset N(\mathscr{B})$, we will concern ourselves in this section with the conditions that ensure inclusions in the other direction. First, however, we present some examples to show that $M(\mathscr{B})$ is indeed a proper subset of $\operatorname{EM}(\mathscr{B})$ and that $E M(\mathscr{B})$ is a proper subset of the set of nonsingular functions, $N(\mathscr{B})$.

The motivation for our first example is as follows: It is clear that if there is to be a function $f$ such that $I(f, \mathscr{B})=0$ but $f \notin E M(\mathscr{B})$, then there must be a sequence $\left\{\left(m_{k}, n_{k}\right)\right\}_{k=1}^{\infty}$ of pairs in $\mathscr{B}$ such that $\alpha\left(m_{k}, n_{k}\right) \neq 0$ but $\alpha\left(m_{k}, n_{k}\right) \rightarrow 0$ as $m_{k} n_{k} \rightarrow \infty$. Obviously, this imposes severe restrictions on $f\left(m_{k}\right), f\left(n_{k}\right)$ and $f\left(m_{k} n_{k}\right)$. If, moreover, $\left(t, m_{k}\right) \in \mathscr{B}$, then $\alpha\left(t, m_{k}\right)$ must be small if $t m_{k}$ is large, and this affects $f(t)$. It seems plausible, then, that a function with the desired properties would be most likely to exist with respect to a basic sequence $\mathscr{B}$ for which the number of integers $t$ such that $\left(t, m_{k}\right) \in \mathscr{B}$ is severely limited. This does not necessitate making $\delta(\mathscr{B})$ small (as we shall see), but can be done by "separating" the pairs $\left(m_{k}, n_{k}\right)$ from the rest of $\mathscr{B}$.

EXAMPle 4.1. Let $p$ and $q$ be distinct primes and let $\mathscr{B}$ be the basic sequence generated by the primitive pair $(p, q)$ and all other primitive pairs in which neither $p$ nor $q$ is a member. In other words, $\mathscr{B}=\Gamma(\mathscr{B}[p, q] \cup(p, p))$, where $\mathscr{B}[p, q]$ was defined in Theorem 3.6. Define $f$ by: $f\left(p^{a}\right)=1+(1 / a)(a \geqq 1), \quad f\left(q^{b}\right)=1+(1 / b)(b \geqq 1)$, $f\left(p^{a} q^{b}\right)=1+(1 / a)+(1 / b)(a \geqq 1, b \geqq 1)$, and $f(n)=1$ otherwise. It is clear that $\alpha(m, n)=0$ if neither $p$ nor $q$ divides $m n$. If $p$ or $q$ does divide $k$, then either $B_{k}$ contains only the trivial pairs $(1, k)$ and $(k, 1)$ or $k=p^{a} q^{b}(a \geqq 1, b \geqq 1)$, and in the latter case $B_{k}$ contains, 
in addition to the two trivial pairs, only the pairs $\left(p^{a}, q^{b}\right)$ and $\left(q^{b}, p^{a}\right)$. Clearly $\alpha\left(p^{a}, q^{b}\right)=(1 / a b) / 2\{1+(1 / a)+(1 / b)+(1 / 2 a b)\}$ which is not zero, but which approaches zero as $p^{a} q^{b} \rightarrow \infty$. Hence $f \in N(\mathscr{B})$ but $f \notin E M(\mathscr{B})$. Finally, we note that

$$
\delta(\mathscr{B}) \geqq \delta(\mathscr{B}[p, q])=\left(1-\frac{1}{p}\right)\left(1-\frac{1}{q}\right)
$$

which can be made arbitrarily close to 1 .

ExAmple 4.2. (a) Let $\mathscr{B}=\mathscr{T}_{2}$ (see Example 3.3). Define $f$ by $f(n)=1$ if $n \neq 2^{a}(a>1), f\left(2^{a}\right)=0$ for $a>1$. Then $\alpha(1, k)=0$ for all $k$ and $\alpha\left(2^{a}, 2^{b}\right)=0$ if $a+b \geqq 3$, so $f \in E M(\mathscr{B})$. But $f(2) f(2) \neq f(4)$, hence $f \notin M(\mathscr{B})$.

(b) Let $\mathscr{B}$ be generated by the pair $(2,3)$ and define $f$ by: $f(n)=n$ if $n \neq 6 ; f(6)=w$ where $w$ is arbitrary except that $w \neq 6$. Then $f \in E M(\mathscr{B}, 7)$ but $f \notin M(\mathscr{B})$.

The failure of each of the functions in Example 4.2 to be multiplicative was due both to the small size of $\mathscr{B}$ and to the values of $k$ for which $f(k)=0$. When $\mathscr{B}$ contains a type II primitive pair, the function in part (a) is in many ways characteristic of functions which are in $E M(\mathscr{B})$ but not in $M(\mathscr{B})$. In fact, when $\mathscr{B}$ is generated by a single type II primitive pair (as is the case here), we shall see from the proof of Theorem 4.5 that we really have very little choice in the construction of $f$. For the time being, however, we note that the zero set $Z_{f}=\{k \mid f(k)=0\}$ in part (a) is small (in fact, $D\left(Z_{f}\right)=0$ ), but that $Z_{f}$ contains all the numbers $2^{t}, t>1$.

The following theorem is an immediate consequence of the definition of nonsingularity.

THEOREM 4.3. If $f$ is nonsingular with respect to $\mathscr{B}$ and if there exists $K>0$ such that either $\alpha(m, n)=0$ or $\beta(m, n)>K$ provided $(m, n) \in \mathscr{B}$ and $m n$ is sufficiently large, then $f \in E M(\mathscr{B})$. In particular, a nonsingular function which assumes only finitely many values is eventually multiplicative with respect to $\mathscr{B}$.

Theorem 4.3 imposes severe demands on $f$ but says little about the basic sequence $\mathscr{B}$. The next lemma, which will be most useful in what follows, depends on the properties of both $f$ and $\mathscr{B}$.

LEMMA 4.4. Let $f$ be a nonsingular function with respect to $\mathscr{B}$ and suppose that $(m, n) \in \mathscr{B}$. If there exists a strictly increasing sequence $\left\{a_{i}\right\}_{i=1}^{\infty}$ of natural numbers such that

(1) $\left(a_{i}, m n\right) \in \mathscr{B}$,

(2) $f\left(a_{i}\right) \neq 0$,

then $f(m n)=f(m) f(n)$. 
Proof. We will consider two cases.

Case 1. $f\left(a_{i} m n\right)=0$ for $a_{i} \geqq N_{1}$. Let $N_{2}$ be the integer given in Lemma 2.3 and let $N=\max \left\{N_{1}, N_{2}\right\}$. If $a_{i} \geqq N$ then

$$
f\left(a_{i}\right) f(m n)=0 \text {; }
$$

hence $f(m n)=0$. Moreover, $f\left(a_{i} n\right) f(m)=0$. If $f(m) \neq 0$, then $f\left(a_{i} n\right)=f\left(a_{i}\right) f(n)=0$; hence $f(n)=0$. Thus $f(m n)=0=f(m) f(n)$.

Case 2. Without loss of generality we may assume $f\left(a_{i} m n\right) \neq 0$ for all $i$. Since $f$ is nonsingular, $J(f)=0$ and $f(1)=1$. It follows from Theorem 2.9 that

$$
f\left(a_{i}\right) f(m n) / f\left(a_{i} m n\right), \quad f\left(a_{i} m\right) f(n) / f\left(a_{i} m n\right),
$$

and $f\left(a_{i}\right) f(m) / f\left(a_{i} m\right)$ all approach 1 as $i \rightarrow \infty$. Hence $f(m n) /$ $f(m) f(n) \rightarrow 1$ and so $f(m) f(n)=f(m n)$.

We note from Example 4.2(a) that Lemma 4.4 is not valid if we omit assumption (2) from the hypothesis.

As an application of the preceding lemma we now prove

THEOREM 4.5. If $f$ is nonsingular with respect to $\mathscr{B}$ and if $(p, p) \in \mathscr{B}$ for some prime $p$, then there is an integer $N_{p}$ such that

$$
f\left(p^{m}\right) f\left(p^{n}\right)=f\left(p^{m+n}\right)
$$

provided $m+n \geqq N_{p}$. If also there is a strictly increasing sequence $\left\{r_{j}\right\}_{j=1}^{\infty}$ of natural numbers such that $f\left(p^{r_{j}}\right) \neq 0$, then $f(p) \neq 0$ and

$$
f\left(p^{n}\right)=f^{n}(p)
$$

for all $n \geqq 0$.

Proof. Let $N$ be the integer given in Lemma 2.3 and suppose $p^{t} \geqq N$. If $f\left(p^{t}\right) \neq 0$, then $f\left(p^{m}\right) f\left(p^{n}\right) \neq 0$ for any non-negative integers $m$ and $n$ such that $m+n=t$. Hence $f\left(p^{m}\right) \neq 0$ for any $0 \leqq m \leqq t$. We conclude that either

(i) there exists an integer $N_{p}$ such that $f\left(p^{t}\right)=0$ for $t \geqq N_{p} / 2$, or

(ii) $f\left(p^{t}\right) \neq 0$ for all $t \geqq 0$.

If case (i) holds, then $f\left(p^{m+n}\right)=0=f\left(p^{m}\right) f\left(p^{n}\right)$ provided $m+n \geqq N_{p}$. On the other hand, if case (ii) holds, we may apply Lemma 4.4 with $a_{i}=p^{i}$, from which it follows that $f\left(p^{m+n}\right)=$ $f\left(p^{m}\right) f\left(p^{n}\right)$ for all $m \geqq 0, n \geqq 0$.

Finally, case (i) cannot hold if $f\left(p^{r_{j}}\right) \neq 0$ for arbitrarily large values of $r_{j}$. That completes the proof. 
COROLlaRY 4.5.1. (a) If $\mathscr{B}$ is generated by a finite set of type II primitive pairs, then $f \in N(\mathscr{B})$ implies $f \in E M(\mathscr{B})$.

(b) Suppose $\mathscr{B}$ is generated by an arbitrary set of type II primitive pairs and $f \in N(\mathscr{B})$. If, for each prime $p$ for which $(p, p) \in \mathscr{B}$, there exists a strictly increasing sequence $\left\{r_{p},{ }_{j}\right\}_{j=1}^{\infty}$ of positive integers such that $f\left(p^{r_{p}, j}\right) \neq 0$, then $f \in M(\mathscr{B})$.

As noted earlier, the zero-set $Z_{f}$ will play an important part in determining whether a function in $\operatorname{EM}(\mathscr{B})$ is also in $M(\mathscr{B})$. Roughly speaking, if $\delta(\mathscr{B})$ is large enough, then for any $(m, n) \in \mathscr{B}$ there will be (many) large values of $t$ for which $(m n, t) \in \mathscr{B}$. Moreover, if $f \in E M(\mathscr{B})$, then $f(m) f(t)=f(m t), f(n) f(t)=f(n t), \quad$ and $f(m n) f(t)=f(m n t)$. Now if $Z_{f}$ is small enough, $f(t), f(m t)$, $f(n t)$, and $f(m n t)$ will be nonzero. Thus $f(m), f(n)$, and $f(m n)$ will be determined by $f(k)$ for large values of $k$, and the multiplicative properties of $f$ for these large values will carry over to $f(m n)$. (The trouble spots in Example 4.2 should now be clear. Even though $D\left(Z_{f}\right)=0$ in part (a), $\mathscr{B}$ was small enough for $f(k)$ to be zero for all $k$ for which $(4, k) \in \mathscr{B}$. And although $Z_{f}=\varnothing$ in part (b), $\mathscr{B}$ was so small then that there simply were no $t$ such that $(6, t) \in \mathscr{B}, t \neq 1)$.

In making the above remarks more precise, the cornerstone of our arguments will be the easily proved

Theorem 4.6. Suppose that $f \in E M(\mathscr{B}, N)$ and let $C_{N}=$ $\Pi_{p<N / 2} p$. If there exists an integer $a \geqq N / 2$ such that

(i) $\left(a, C_{N}\right) \in \mathscr{B}$,

(ii) $f(a) \neq 0$, then $f$ is multiplicative with respect to $\mathscr{B}$.

Proof. Choose $m$ and $n$ such that $m n<N,(m, n) \in \mathscr{B}$. Since $f(1)=1$, we may assume $m>1$ and $n>1$; therefore $m<N / 2$ and $n<N / 2$ and we may decompose $m n$ canonically: $m n=q_{1}^{\gamma_{1}} q_{2}^{\gamma_{2}} \cdots q_{r}^{\gamma_{r}}$, where each $q_{i}$ is a prime less than $N / 2$. Thus each $q_{i} \mid C_{N}$.

Since $\left(a, C_{N}\right) \in \mathscr{B}$, we have $\left(a, q_{i}\right) \in \mathscr{B}, 1 \leqq i \leqq r$; hence $(a, m n)$, $(a, m),(a, n)$, and $(a m, n)$ are all in $\mathscr{B}$. Finally, since $f \in E M(\mathscr{B}, N)$ and $a m n>a m \geqq N$, we have

$$
f(a) f(m n)=f(a m n)=f(a m) f(n)=f(a) f(m) f(n) .
$$

Thus $f(m n)=f(m) f(n)$ and the proof is complete.

The following corollary and the next three theorems all depend on Theorem 4.6. They illustrate the remarks preceding that theorem and emphasize the importance of the relationship between $Z_{f}$ and $\mathscr{B}$. 
Corollary 4.6.1. Suppose $f \in E M(\mathscr{B}, N)$ and, as before, let $C_{N}=\Pi_{p<N / 2} p$. Also let $A=\left\{a \mid\left(a, C_{N}\right) \in \mathscr{B}\right\}$. If

(1) $\underline{D}(A)=\theta>0$

(2) $\bar{D}\left(Z_{f}\right)<\theta$, then $f$ is multiplicative with respect to $\mathscr{B}$.

Proof. Let $\eta=\left(\theta-\bar{D}\left(Z_{f}\right)\right) / 3>0$ and choose $K$ such that $x>K$ implies

$$
\frac{\# Z_{f}(x)}{x}<\bar{D}\left(Z_{f}\right)+\eta<\theta-\eta<\frac{\# A(x)}{x} .
$$

Then ${ }^{\sharp} A(x)-{ }^{\sharp} Z_{f}(x)>\left(\theta-\bar{D}\left(Z_{f}\right)-2 \eta\right) x=\eta x$ provided $x>K$. Thus ${ }^{\sharp} A(x)-{ }^{\sharp} Z_{f}(x) \rightarrow \infty$ as $x \rightarrow \infty$, and the corollary follows.

THEOREM 4.7. Suppose $f \in E M(\mathscr{B}, N)$ and that

$$
\bar{\delta}(\mathscr{B}) \geqq 1-\lambda_{M},
$$

where $M=[N / 2]+1$ and $\lambda_{M}$ is as defined in Theorem 3.7. If $p_{1}, \cdots, p_{K}$ are the primes less than $N / 2$, then $f \in M(\mathscr{B})$ provided

$$
\liminf _{n \rightarrow \infty} \frac{\# Z_{f}(n)}{\log ^{K}(n / M)}<\frac{1}{K !\left(\log p_{1} \cdots \log p_{K}\right)} .
$$

Proof. Assume $N \geqq 5$, since otherwise $\operatorname{EM}(\mathscr{B}, N)$ and $M(\mathscr{B})$ are identical. If $(M, p) \notin \mathscr{B}$ for a prime $p<N / 2$, then $(q, p) \notin \mathscr{B}$ for some prime $q \leqq M$. It is a simple exercise to show that, for fixed $y \geqq 2$, the function

$$
F(x)=1-\frac{1}{2}\left(\lambda_{y}+\frac{1}{y}\right)\left(\lambda_{x}+\frac{1}{x}\right)+\lambda_{y} \lambda_{x}
$$

increases for $x \geqq 2$, and it is also easy to show that

$$
1-\frac{1}{2}\left(\lambda_{x}+\frac{1}{x}\right)^{2}+\lambda_{x}^{2}<1-\lambda_{x}
$$

for $x \geqq 2$. Therefore, if $p \neq q$, the above and Remark 3.8 yield

$$
\begin{aligned}
\bar{\delta}(\mathscr{B}) & \leqq 1-\frac{1}{2}\left(\lambda_{p}+\frac{1}{p}\right)\left(\lambda_{q}+\frac{1}{q}\right)+\lambda_{p} \lambda_{q} \\
& <1-\frac{1}{2}\left(\lambda_{M}+\frac{1}{M}\right)^{2}+\lambda_{M}^{2}<1-\lambda_{M} .
\end{aligned}
$$

If, on the other hand, $p=q$, Theorem 3.7 gives

$$
\bar{\delta}(\mathscr{B}) \leqq 1-\lambda_{p}<1-\lambda_{M} \text {. }
$$


In either case, $\bar{\delta}(\mathscr{B})<1-\lambda_{M}$, which contradicts the hypothesis. Hence $(M, p) \in \mathscr{B}$ for every prime $p<N / 2$ and so $\left(M, C_{N}\right) \in \mathscr{B}$.

Suppose now that $f \notin M(\mathscr{B})$. Then, by Theorem 4.6, $f(M)=$ 0 . If $t \geqq 2$ is divisible only by primes $p<N / 2$, then, by the above, $(t, M) \in \mathscr{B}$, and so $f(t M)=f(t) f(M)=0$. Since there are

$$
\frac{\log ^{K}(n / M)}{K !\left(\log p_{1} \cdots \log p_{K}\right)}+O\left(\log ^{K-1}(n / M)\right)
$$

integers $t \leqq n / M$ divisible only by $p_{1}, \cdots, p_{K}$, there are at least that many integers $k \leqq n$ such that $f(k)=0$. Therefore,

$$
\liminf _{n \rightarrow \infty} \frac{\# Z_{f}(n)}{\log ^{K}(n / M)} \geqq \frac{1}{K !\left(\log p_{1} \cdots \log p_{K}\right)},
$$

and the proof is complete.

If we know something about the structure of the basic sequence $\mathscr{B}$, we may be able to weaken the conditions of the preceding theorem. For instance, recalling from Example 3.1 that $\mathscr{C}$ is the basic sequence consisting of all pairs $(a, b)$ such that $a$ and $b$ are relatively prime, we have

THEOREM 4.8. If $D\left(Z_{f}\right)=0$, then $f \in E M(\mathscr{C})$ implies $f \in M(\mathscr{M})$.

Proof. Suppose that $f \in E M(\mathscr{C l}, N)$ and $A=\left\{a \mid\left(a, C_{N}\right) \in \mathscr{L}\right\}$, with $C_{N}$ again as given in Theorem 4.6. Then $a \in A$ if and only if $a$ is not divisible by any prime $p<N / 2$. Now it is easy to show that $D(A)=\prod_{p<N / 2}\{1-(1 / p)\}$ and, since $\bar{D}\left(Z_{f}\right)=0<D(A)$, the conclusion follows from Corollary 4.6.1.

THEOREM 4.9. If $f \in E M(\mathscr{B}, N)$ and $\bar{\delta}(\mathscr{B})=1$, then $f$ is a character.

Proof. Choose $a \geqq N / 2$ such that $f(a) \neq 0$. By Theorem 3.9, $\mathscr{B}=\mathscr{L}$; hence $(a, p) \in \mathscr{B}$ for every $p<N / 2$. It follows now from Theorem 4.6 that $f \in M(\mathscr{L})$.

In anticipation of its importance in connection with singular functions, we close this section with a short discussion about a particular type of nonsingular function: An arithmetic function $f$ will be called a unit with respect to $\mathscr{B}$ if (i) $f \in N(\mathscr{B})$ and (ii) there exists an integer $N$ such that $f(k)$ is not zero and does not change sign for $k \geqq N$. A unit $f$ will be called positive or negative depend- 
ing on the sign of $f(k)$ for large values of $k$.

From Lemma 2.3 we may easily prove

Lemma 4.10. If there is an integer $N$ such that

(1) $f(k)<0$ for $k \geqq N$,

(2) $\bar{\beta}(f, \mathscr{B})<1$,

then $\mathscr{B}=\mathscr{S}$. In particular, negative units can exist only with respect to $\mathscr{S}$.

A straightforward application of Lemma 4.4 yields the following useful theorem on units.

THEOREM 4.11. (a) Suppose $f$ is a unit with respect to $\mathscr{B}$ and $(m, n) \in \mathscr{B}$. If there is an integer $a>1$ such that $(a, m n) \in \mathscr{B}$, then

(1) $f(m)>0, f(n)>0$,

(2) $f(m n)=f(m) f(n)$.

(b) If $f$ is a unit with respect to $\mathscr{B}$ and $\bar{\delta}(\mathscr{B})=1$, then

(1) $f(k)>0$ for all $k$,

(2) $f$ is a character.

(c) If $f$ is a unit with respect to $\mathscr{C l}$, then

(1) $f(k)>0$ for all $k$,

(2) $f$ is multiplicative with respect to $\mathscr{K}$.

Finally, from Corollary 4.5.1 we have

THEOREM 4.12. If $\mathscr{B}$ is generated by an arbitrary collection of type II primitive pairs and if $f$ is a unit with respect to $\mathscr{B}$, then $f$ is multiplicative with respect to $\mathscr{B}$.

5. Singular functions. We conclude this paper with a discussion of those singular functions for which $I(f, \mathscr{B})$ exists. As the following theorem shows, these functions are closely connected with the set of units on $\mathscr{B}$.

THEOREM 5.1. Let an arithmetic function $f$ and a basic sequence $\mathscr{B}$ be given. Then $I(f, \mathscr{B})$ exists and $0<|I(f, \mathscr{B})|<1$ if and only if

$$
f=M f^{*},
$$

where (i) $f^{*}$ is a unit with respect to $\mathscr{B}$,

(ii) $M>0, M \neq 1$.

Moreover, the decomposition $f=M f^{*}$ is unique. 
Proof. The uniqueness of the decomposition described above, if such a decomposition exists, is immediate. For suppose

$$
0<|I(f, \mathscr{B})|<1 \text { and } f=M f^{*} \text {, }
$$

where $f^{*}$ is a unit with respect to $\mathscr{B}$. Then

$$
f(1)=M f^{*}(1)=M \text { and } f^{*}(k)=f(k) / f(1) .
$$

Assume now that $0<|I(f, \mathscr{B})|<1$. We know from Lemma 2.4 that $f(1)>0$ and from Theorem 2.5 that $f(1) \neq 1$. Let $M=$ $f(1)$ and let $f^{*}(k)=f(k) / f(1)$. Again by Theorem 2.5, $f(k)$ does not change sign for sufficiently large $k$ and so $f^{*}(k)$ has the same property.

If $\mathscr{B}=\mathscr{S}$ it follows from the above that $I\left(f^{*}, \mathscr{S}\right)$ exists and, since $f^{*}(1)=1, f^{*}$ is a unit with respect to $\mathscr{S}$.

If $\mathscr{B} \neq \mathscr{S}$, Corollary 2.6.1 implies $f(k)>0$ for all sufficiently large $k$, hence $I(f, \mathscr{B})=(f(1)-1) /(f(1)+1)$. Therefore, from Lemma 2.3, we have

$$
\frac{f(1)-1}{f(1)+1}-\varepsilon<\frac{\frac{f(m) f(n)}{f(m n)}-1}{\frac{f(m) f(n)}{f(m n)}+1}<\frac{f(1)-1}{f(1)+1}+\varepsilon
$$

whenever $(m, n) \in \mathscr{B}, m n \geqq N(\varepsilon)$. Consequently, if $\rho_{\nu}$ is defined by (2.3), then $\rho_{\nu} \rightarrow f(1)$. But then if $\rho_{\nu}^{*}=f^{*}\left(m_{\nu}\right) f^{*}\left(n_{\nu}\right) / f^{*}\left(m_{\nu} n_{\nu}\right)$, $\rho_{\nu}^{*} \rightarrow 1$. It follows from Theorem 2.9 that $J\left(f^{*}, \mathscr{B}\right)$ exists. Finally, since $f^{*}(1)=1, J\left(f^{*}, \mathscr{B}\right)=I\left(f^{*}, \mathscr{B}\right)=0$.

Conversely, suppose $f=M f^{*}$, where $M>0, M \neq 1, f^{*}$ a unit with respect to $\mathscr{B}$. Since $f(k)$ does not change sign for all sufficiently large $k, I(f, \mathscr{S})$ exists. By Theorem $2.5,0<|I(f, \mathscr{S})|<1$. If $\mathscr{B} \neq \mathscr{S}$, it follows from $J\left(f^{*}, \mathscr{B}\right)=0$ (and so $f^{*}(1)=1$ ) and Theorem 2.9 that $\rho_{\nu}^{*} \equiv f^{*}\left(m_{\nu}\right) f^{*}\left(n_{\nu}\right) / f^{*}\left(m_{\nu} n_{\nu}\right) \rightarrow 1$ when

$$
\left(m_{\nu}, n_{\nu}\right) \in \mathscr{B}, \quad m_{\nu} n_{\nu} \rightarrow \infty, \quad f^{*}\left(m_{\nu} n_{\nu}\right) \neq 0 .
$$

But then if $\rho_{\nu}$ satisfies (2.3), $\rho_{\nu}=M \rho_{\nu}^{*} \rightarrow M=f(1)$. Therefore, by Theorem 2.9, $J(f, \mathscr{B})$ exists. Since $0<J(f, \mathscr{B})<1$ (Theorem 2.5) and since $f(k)>0$ for all sufficiently large $k$ ( $f^{*}$ must be a positive unit), we have with the aid of Lemma 2.3

$$
\alpha_{f}\left(m_{\nu}, n_{\nu}\right)=\frac{\rho_{\nu}-1}{\rho_{\nu}+1} \rightarrow \frac{M-1}{M+1} .
$$

Thus $I(f, \mathscr{B})$ exists and $0<|I(f, \mathscr{B})|<1$.

A partial analogue to Theorem 5.1 will hold when $I(f, \mathscr{B})= \pm 1$. The proof is straightforward and is omitted. 
THEOREM 5.2. (a) If $\mathscr{B}=\mathscr{S}$ and $f(1) \neq 0$, then $I(f, \mathscr{B})$ exists and has the value \pm 1 if and only if $f=M f^{*}$, where $f^{*}$ is a unit with respect to $\mathscr{S}$ and $M<0$. Moreover, the decomposition is unique.

(b) If $\mathscr{B} \neq \mathscr{S}$ and $f=M f^{*}$, where $f^{*}$ is a unit with respect to $\mathscr{B}$ and $M<0$, then $I(f, \mathscr{B})$ exists and has the value +1 .

EXAMPLE 5.3. (a) If $I(f)=+1$ then $f^{*} \equiv f / f(1)$ may or may not be a unit. For example, let $\mathscr{B}=\Gamma((2,2)), f(1)=-1$, $f\left(2^{a}\right)=-k$ where $k>0, k \neq 1, a>0$, and let $f(t)$ be any negative number otherwise. Then $I\left(f^{*}, \mathscr{B}\right)$ does not exist. On the other hand, let $\mathscr{B}$ be arbitrary and let $f(k)=-1$ for all $k$. Then $f^{*}$ is a unit with respect to $\mathscr{B}$.

(b) If $I(f, \mathscr{B})=-1$ and $\mathscr{B} \neq \mathscr{S}$, then $f(k)>0$ for sufficiently large $k$ and $f(1) \leqq 0$. Hence (assuming $f(1)<0) f^{*}(k) \equiv$ $f(k) / f(1)<0$ for large $k$ and so $f^{*}$ can never be a unit with respect to $\mathscr{B}$.

Although, as the preceding example shows, it is not usually possible to express a function $f$ for which $I(f)=-1$ as a multiple of a unit, we can characterize such functions in terms of the behavior of $f(m) f(n) / f(m n)$.

THEOREM 5.4. I $(f, \mathscr{B})$ exists and has the value -1 if and only if

(1) $f(1) \leqq 0$,

(2) there is an integer $N$ such that

(a) $f(k)>0$ for $k \geqq N$,

(b) $f(m) f(n) / f(m n) \rightarrow 0$ as $m n \rightarrow \infty,(m, n) \in \mathscr{B}, m \geqq N$, $n \geqq N$,

(c) if $1<m_{0}<N$ and $f\left(m_{0}\right)>0$, then $f(n) / f\left(m_{0} n\right) \rightarrow 0$ as $n \rightarrow \infty,\left(m_{0}, n\right) \in \mathscr{B}$.

Proof. If $I(f, \mathscr{B})=-1$, parts (1) and (2a) follow from Lemma 2.4. Parts $(2 \mathrm{~b})$ and (2c) are easy consequences of part (2a) and definition (2.1). The converse also follows readily from definition (2.1).

The following example shows that (2c) may not be omitted from the above.

ExAmple 5.5. Let $\mathscr{B}=\Gamma((2,3))$. Define $f(k)=1$ if $k=2$, $3^{n}$, or $2 \cdot 3^{n}$ for $n \geqq 1 ; f(k)=-1$ if $k=1$ or $4 ; f(k)=k^{k}$ otherwise. Conditions (1), (2a), and (2b) are satisfied; (2c) is not. $I(f, \mathscr{B})$ does not exist.

Theorem 5.1 has an interesting application with regard to the index 
of a product of two arithmetic functions. First we need a lemma.

LEMMA 5.6. If $f$ and $g$ are units with respect to $\mathscr{B}$, then $f g$ is also a unit with respect to $\mathscr{B}$.

Proof. The lemma follows readily from Theorem 2.9.

Suppose now that either (i) $f$ is a unit with respect to $\mathscr{B}$ or (ii) $0<|I(f, \mathscr{B})|<1$. Then $f(k)$ does not change sign for sufficiently large $k$, and it is useful to define a function $\omega$ by: $\omega(f)=$ +1 if $f(k)>0$ for all large $k$ and $\omega(f)=-1$ if $f(k)<0$ for large $k$.

THEOREM 5.7. Suppose that $f$ and $g$ are arithmetic functions such that either $f$ is a unit with respect to $\mathscr{B}$ or $0<|I(f, \mathscr{B})|<1$, and similarly for $g$. Then $I(f g, \mathscr{B})$ exists and

$$
I(f g)=\omega(f) \omega(g) \frac{\omega(f) I(f)+\omega(g) I(g)}{1+\omega(f) \omega(g) I(f) I(g)} .
$$

In particular, if $\mathscr{B} \neq \mathscr{S}$,

$$
I(f g)=\frac{I(f)+I(g)}{1+I(f) I(g)}
$$

Proof. The theorem is easily proved by considering the two cases $\mathscr{B}=\mathscr{S}$ and $\mathscr{B} \neq \mathscr{S}$ and, within each case, by considering the various combinations of $f$ and $g$. We illustrate one of the possibilities:

Suppose $\mathscr{B} \neq \mathscr{S}, 0<|I(f, \mathscr{B})|<1$, and $0<|I(g, \mathscr{B})|<1$. From Theorem 5.1 we know that $f=M f^{*}$ and $g=K g^{*}$, where $f^{*}$ and $g^{*}$ are units, $M>0, M \neq 1, K>0, K \neq 1$. Therefore $f g=M K\left(f^{*} g^{*}\right)$ and, by Lemma 5.6, $f^{*} g^{*}$ is also a unit with respect to $\mathscr{B}$. Since

$$
I(f)=(M-1) /(M+1), \quad I(g)=(K-1) /(K+1),
$$

and $I(f g)=(M K-1) /(M K+1)$, we have

$$
M=(1+I(f)) /(1-I(f))
$$

and $K=(1+I(g)) /(1-I(g))$, and the desired relation follows if we substitute these values in the expression for $I(f g)$.

Corollary 5.7.1. Suppose $\mathscr{B} \neq \mathscr{S}$. If $0<|I(f, \mathscr{B})|<1$ and $I(g, \mathscr{B})=-I(f, \mathscr{B})$, then $f g$ is a unit with respect to $\mathscr{B}$.

The extension from the product of two functions to the product 
of any finite number of arithmetic functions is easily accomplished by induction. For let $\sigma_{m}\left[x_{1}, \cdots, x_{n}\right]$ be the $m$-th elementary symmetric function of the variables $x_{1}, \cdots, x_{n}$. That is, $\sigma_{0}\left[x_{1}, \cdots, x_{n}\right]=1$ and $\sigma_{m}\left[x_{1}, \cdots, x_{n}\right]=\sum_{1 \leqq i_{1}<\cdots<i_{m} \leqq n} x_{i_{1}} \cdots x_{i_{m}}$ for $1 \leqq m \leqq n$. Then we have

THEOREM 5.8. Let $f_{1}, f_{2}, \cdots, f_{n}$ be arithmetic functions such that, for each $1 \leqq i \leqq n$, either $f_{i}$ is a unit with respect to $\mathscr{B}$ or $0<\left|I\left(f_{i}, \mathscr{B}\right)\right|<1$. Also, for $0 \leqq m \leqq n$, let

$$
\sigma_{m}^{n}=\sigma_{m}\left[\omega\left(f_{1}\right) I\left(f_{1}\right), \cdots, \omega\left(f_{n}\right) I\left(f_{n}\right)\right] .
$$

Then $I\left(f_{1} \cdots f_{n}\right)$ exists and

$$
I\left(f_{1} \cdots f_{n}\right)=\left\{\begin{array}{c}
\omega\left(f_{1}\right) \cdots \omega\left(f_{n}\right) \frac{\sigma_{1}^{n}+\sigma_{3}^{n}+\cdots+\sigma_{n-1}^{n}}{\sigma_{0}^{n}+\sigma_{2}^{n}+\cdots+\sigma_{n}^{n}} \\
\text { if } n \text { is even }, \\
\omega\left(f_{1}\right) \cdots \omega\left(f_{n}\right) \frac{\sigma_{1}^{n}+\sigma_{3}^{n}+\cdots+\sigma_{n}^{n}}{\sigma_{0}^{n}+\sigma_{2}^{n}+\cdots+\sigma_{n-1}^{n}} \\
\text { if } n \text { is odd. }
\end{array}\right.
$$

I would like to thank Professors Michael Aissen and Hing Tong for their valuable suggestions and Mr. Benedict Scott for programming the computation used in Theorem 3.10 to evaluate $\delta(\mathscr{C l})$.

\section{BIBLIOGRAPHY}

1. G. H. Hardy and E. M. Wright, An Introduction to the Theory of Numbers, Fourth Edition, Oxford, 1960.

2. I. Niven and H.S. Zuckerman An Introduction to the Theory of Numbers, Wiley, New York, 1960.

Received January 5, 1967, and in revised form May 5, 1967. This research was supported in part by the National Science Foundation under grant GP-6590.

FORDHAM UNIVERSITY

BRONX, NEW YORK 


\section{PACIFIC JOURNAL OF MATHEMATICS}

\section{EDITORS}

\section{H. ROYDEN}

Stanford University

Stanford, California

\author{
R. R. Phelps \\ University of Washington \\ Seattle, Washington 98105
}

\section{J. DugundJI}

Department of Mathematics University of Southern California Los Angeles, California 90007

\section{RICHARD ARENS}

University of California

Los Angeles, California 90024

\section{ASSOCIATE EDITORS}

\section{E. F. BECKENBACH}

B. H. NEUMANN

F. WOLF

K. YOSIDA

\section{SUPPORTING INSTITUTIONS}

UNIVERSITY OF BRITISH COLUMBIA CALIFORNIA INSTITUTE OF TECHNOLOGY UNIVERSITY OF CALIFORNIA MONTANA STATE UNIVERSITY UNIVERSITY OF NEVADA NEW MEXICO STATE UNIVERSITY OREGON STATE UNIVERSITY UNIVERSITY OF OREGON OSAKA UNIVERSITY UNIVERSITY OF SOUTHERN CALIFORNIA

\author{
STANFORD UNIVERSITY \\ UNIVERSITY OF TOKYO \\ UNIVERSITY OF UTAH \\ WASHINGTON STATE UNIVERSITY \\ UNIVERSITY OF WASHINGTON \\ AMERICAN MATHEMATICAL SOCIETY \\ CHEVRON RESEARCH CORPORATION \\ TRW SYSTEMS
}

NAVAL WEAPONS CENTER

Mathematical papers intended for publication in the Pacific Journal of Mathematics should be in typed form or offset-reproduced, double spaced with large margins. Underline Greek letters in red, German in green, and script in blue. The first paragraph or two must be capable of being used separately as a synopsis of the entire paper. It should not contain references to the bibliography. Manuscripts, in duplicate if possible, may be sent to any one of the four editors. All other communications to the editors should be addressed to the managing editor, Richard Arens, University of California, Los Angeles, California 90024.

Each author of each article receives 50 reprints free of charge; additional copies may be obtained at cost in multiples of 50 .

The Pacific Journal of Mathematics is published monthly. Effective with Volume 16 the price per volume (3 numbers) is $\$ 8.00$; single issues, $\$ 3.00$. Special price for current issues to individual faculty members of supporting institutions and to individual members of the American Mathematical Society: $\$ 4.00$ per volume; single issues $\$ 1.50$. Back numbers are available.

Subscriptions, orders for back numbers, and changes of address should be sent to Pacific Journal of Mathematics, 103 Highland Boulevard, Berkeley 8, California.

Printed at Kokusai Bunken Insatsusha (International Academic Printing Co., Ltd.), 7-17, Fujimi 2-chome, Chiyoda-ku, Tokyo, Japan.

PUBLISHED BY PACIFIC JOURNAL OF MATHEMATICS, A NON-PROFIT CORPORATION

The Supporting Institutions listed above contribute to the cost of publication of this Journal, but they are not owners of publishers and have no responsibility for its content or policies. 


\section{Pacific Journal of Mathematics}

\section{Vol. 27, No. $2 \quad$ February, 1968}

Leonard E. Baum and George Roger Sell, Growth transformations for

functions on manifolds ............................ 211

Henry Gilbert Bray, A note on CLT groups ................... 229

Paul Robert Chernoff, Richard Anthony Rasala and William Charles

Waterhouse, The Stone-Weierstrass theorem for valuable fields....... 233

Douglas Napier Clark, On matrices associated with generalized

interpolation problems ................................

Richard Brian Darst and Euline Irwin Green, On a Radon-Nikodym theorem for finitely additive set functions . ...................... 255

Carl Louis DeVito, A note on Eberlein's theorem..................... 261

P. H. Doyle, III and John Gilbert Hocking, Proving that wild cells exist . . . 265

Leslie C. Glaser, Uncountably many almost polyhedral wild $(k-2)$-cells in

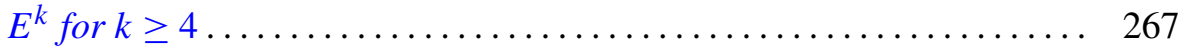

Samuel Irving Goldberg, Totally geodesic hypersurfaces of Kaehler

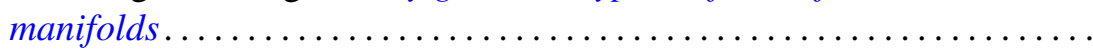

Donald Goldsmith, On the multiplicative properties of arithmetic functions .................................... 283

Jack D. Gray, Local analytic extensions of the resolvent ............ 305

Eugene Carlyle Johnsen, David Lewis Outcalt and Adil Mohamed Yaqub,

Commutativity theorems for nonassociative rings with a finite division ring homomorphic image ....................

André (Piotrowsky) De Korvin, Normal expectations in von Neumann

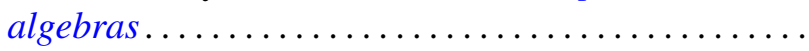

James Donald Kuelbs, A linear transformation theorem for analytic

Feynman integrals..........................

W. Kuich, Quasi-block-stochastic matrices ................... 353

Richard G. Levin, On commutative, nonpotent archimedean

semigroups ............................... 365

James R. McLaughlin, Functions represented by Rademacher series ... . . . 373

Calvin R. Putnam, Singular integrals and positive kernels............ 379

Harold G. Rutherford, II, Characterizing primes in some noncommutative

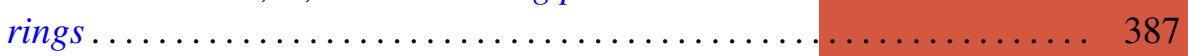

Benjamin L. Schwartz, On interchange graphs................... 393

Satish Shirali, On the Jordan structure of complex Banach *algebras . . . . . 397

Earl J. Taft, A counter-example to a fixed point conjecture............. 405

J. Roger Teller, On abelian pseudo lattice ordered groups ..... 